\title{
Early acute hepatitis with parenteral amiodarone: a toxic effect of the vehicle?
}

\author{
A Rhodes, J B Eastwood, S A Smith
}

\begin{abstract}
A 72 year old white man developed acute hepatic impairment and renal failure within 24 hours of starting intravenous amiodarone for paroxysmal ventricular tachycardia. After normal initial investigations, there was a noticeable rise in serum transaminases as well as an increase in clotting times, a decrease in renal function and a thrombocytopenia. These changes returned to normal within seven days of withdrawal of the drug without specific treatment, and the patient was later treated with oral amiodarone without any further evidence of hepatotoxicity. Intravenous amiodarone has been implicated in acute hepatic disease on four previous occasions, but it is suggested that polysorbate 80 , an organic surfactant added to the intravenous infusion, is a more likely cause of this complication. Similar reactions have been described with polysorbate 80 in association with the 'E-ferol' syndrome in infants. The occurrence of acute hepatic impairment with intravenous amiodarone does not necessarily preclude the use of this drug by mouth.

(Gut 1993; 34: 565-566)
\end{abstract}

A 72 year old man with ischaemic heart disease and poor ventricular function was admitted to hospital after collapsing at home. He had previously had three vessel coronary bypass grafts in 1977 and then had a myocardial infarction after cardiac catheterisation for unstable angina in April 1991.

At the time of admission he was in mild congestive cardiac failure, with a mildly raised jugular venour pressure at $3 \mathrm{~cm}$ but no other evidence of oedema, ascites, or hepatomegaly. His electrocardiogram showed sinus rhythm with widespread ST-T wave abnormalities. He was on long term treatment with frusemide ( $80 \mathrm{mg}$ twice a day), enalapril (10 $\mathrm{mg}$ once a day), isosorbide mononitrate (10 $\mathrm{mg}$ once a day), and aspirin (150 mg once a day). These drugs had not been changed over the previous three months. During the first 24 hours in hospital, he had three sustained episodes of ventricular tachycardia, at a rate of 200 per minute, associated with circulatory collapse and syncope. All three episodes were self terminating after 1-4 minutes. He was started on intravenous amiodarone at a rate of $300 \mathrm{mg}$ over the first hour followed by 900 mg over the next 24 hours.

The results of initial laboratory investigations were normal for hepatic, renal (Table), and thyroid function but there was mild hypokalaemia for which supplementation was started with Sando-K (two tablets three times a day). Within 12 hours of starting the intravenous amiodarone the liver function tests were noticeably abnormal and he had developed signs of hepatic encephalopathy (grade 1). He became oliguric with increases in creatinine and urea. There was a neutrophil leukocytosis with appreciable derangement of the clotting times. He subsequently became thrombocytopenic with hypokalaemia and hypocalcaemia.

A toxic hepatitis was suspected and the amiodarone was stopped after 24 hours. The hepatitis and the renal failure were treated conservatively. Fresh frozen plasma was given to correct the clotting. Within 48 hours there was a symptomatic improvement and the chemical and haematological abnormalities had begun to improve.

At day 7 oral amiodarone $(200 \mathrm{mg}$ three times daily) was reintroduced with no adverse effects. The liver function continued to improve. There was no recurrence of his ventricular tachycardias
St George's Hospital, Department of Medicine, Tooting, London A Rhodes J B Eastwood

S A Smith

Correspondence to: Dr A Rhodes, Farthings, Chapel Lane, Curdridge, Southampton SO3 2BB. Accepted for publication 1 September 1992
Patient's biochemical and haematological parameters over the course of the hospital admission *

\begin{tabular}{|c|c|c|c|c|c|c|c|c|c|c|}
\hline Day & Treatment & $\begin{array}{l}C \\
(\mu \mathrm{mol} / \mathrm{l})\end{array}$ & $\begin{array}{l}B \\
(\mu \mathrm{mol} / \mathrm{l})\end{array}$ & $\begin{array}{l}A L T \\
U / l\end{array}$ & $\underset{U / l}{A S T}$ & $\begin{array}{l}L D H \\
U / l\end{array}$ & $\begin{array}{l}P L T \\
\left(\times 10^{9} / l\right)\end{array}$ & $\begin{array}{l}P T \\
(s)\end{array}$ & $\begin{array}{l}W T \\
(k g)\end{array}$ & $\begin{array}{l}\text { Urine } \\
\text { vol }(m l)\end{array}$ \\
\hline 1 & Admitted & 134 & 21 & 69 & $\begin{array}{l}50 \\
50\end{array}$ & 167 & & & 70 & \\
\hline 2 & Amiodarone iv & 140 & 27 & 84 & 59 & 192 & & & 72 & \\
\hline 3 & Amiodarone iv & 272 & 71 & 4642 & 5236 & 9262 & 204 & 34 & 73 & 150 \\
\hline 4 & No treatment & 328 & 63 & 7238 & & 6204 & 147 & 35 & 73 & 1015 \\
\hline 5 & No treatment & 264 & & & & & 98 & 23 & 72 & 1770 \\
\hline 6 & No treatment & & & & & & 92 & 14 & 71 & 3000 \\
\hline 7 & No treatment & 132 & 64 & 2695 & 337 & 220 & 106 & & 70 & 3850 \\
\hline 8 & No treatment & 113 & 50 & 1915 & 196 & 177 & 124 & & 69 & 4450 \\
\hline 9 & Amiodarone po & 112 & 36 & 1360 & & & 113 & & 70 & 3000 \\
\hline 10 & Amiodarone po & 120 & 33 & & & & 145 & & & 3000 \\
\hline 13 & Amiodarone po & 143 & 25 & 478 & & & 84 & & & \\
\hline 14 & Amiodarone po & 135 & 19 & 322 & & & 156 & & & \\
\hline 15 & Amiodarone po & 132 & 20 & 289 & & & 176 & & & \\
\hline 16 & Amiodarone po & 145 & 19 & & & & 175 & & & \\
\hline \multicolumn{2}{|c|}{ al ranges } & $(60-130)$ & $(0-20)$ & $(0-45)$ & $(0-40)$ & $(6-225)$ & $(150-400)$ & $(11-15)$ & & \\
\hline
\end{tabular}

${ }^{\star} \mathrm{LDH}$ isoenzymes unavailable

$\mathrm{C}=$ creatinine; $\mathrm{B}=$ bilirubin; $\mathrm{ALT}=$ alanine aminotransferase; $\mathrm{AST}=$ aspartate aminotransferase; $\mathrm{LDH}=$ lactate dehydrogenase PLT = platelet count PT $=$ prothrombin time; WT = weight Urine $=$ volume of urine in 24 hours

Doses of amiodarone, iv; $300 \mathrm{mg}$ over 1 hour followed by $900 \mathrm{mg}$ over 24 hours: amiodarone, po; $200 \mathrm{mg}$ three times a day. 
while he remained in hospital. The patient was discharged home 17 days after admission with no further arrythmias or biochemical dysfunction.

He went home feeling better than he had done for some time but he died suddenly during sleep three days later, presumably from a breakthrough cardiac arrythmia. All other drugs, with the exception of Sando-K had continued unchanged throughout.

\section{Discussion}

Amiodarone is an iodinated benzofurane derivative which is used in a wide variety of cardiac arrythmias resistant to other treatments. It has a long half life and may be administered either orally or intravenously. It has the benefit of having no negative inotropic properties.

The drug has many extracardiac side effects, ${ }^{1}$ including corneal microdeposits, photosensitivity, and hypo- or hyperthyroidism. Some 15$40 \%$ of patients taking it long term develop an asymptomatic hepatic dysfunction, which is both dose dependent and reversible on stopping treatment. ${ }^{23}$

A similar biochemical picture to this may be seen with severe heart failure. It is unlikely, however, that the rises seen in the alanine aminotransferase, aspartate aminotransferase, and lactate dehydrogenase activities in this patient have anything other than hepatic cause. The biochemical test to prove this would have been the differentiation between the cardiac and the hepatic lactate dehydrogenase isoenzymes. Unfortunately, these were not available.

Severe acute hepatitis immediately after intravenous amiodarone has been reported four times. ${ }^{+6}$ Never before, however, has oral amiodarone been reintroduced after this type of reaction. As oral amiodarone had no adverse effect on the liver function in our patient, we suggest that it may have been one of the other constituents of the intravenous solution which was responsible for the hepatotoxicity.

In order to obtain stable solutions of amiodarone for intravenous use, the drug is dissolved in a mixture of polysorbate 80 (polyoxenethylated sorbitan ester) a small amount of benzylacohol, and water. Polysorbate 80 has been implicated in the E-ferol syndrome which has been described in infants. ${ }^{?}$

The E-ferol syndrome is characterised by hepatomegaly, splenomegaly, cholestatic jaundice, renal failure, and thrombocytopenia. It is associated with the use of an intravenous preparation of vitamin E, E-ferol, which contains polysorbate 80 and polysorbate 20 . The liver histology in this syndrome shows Kupffer cell exfoliation, centrilobular accumulation of cellular debris, and panlobular congestion, especially in central areas. The polysorbates are deemed responsible for these changes. The clinical features of the E-ferol syndrome show noticeable similarities to those found in our cases of acute hepatitis after intravenous amiodarone and similar cases described by others.

Although this particular adverse reaction of intravenous amiodarone is rare, it remains important because of the popularity of amiodarone for the treatment of severe life threatening cardiac arrythmias.

Amiodarone, with its lack of negative inotropism, broad spectrum of action, and high efficacy is the only drug of its kind available for use in life threatening arrythmias in patients with severe left ventricular impairment. We feel that the occurrence of acute hepatitis with the intravenous preparation does not necessarily preclude the subsequent use of the oral preparation in these patients. We would advocate caution, however, and recommend careful monitoring of hepatic and renal parameters.

This case has been reported to the Committee of Safety of Medicines.

1 Harris L, McKenna W, Rowland E, Storey G, Krikler D. Side effects of long term amiodarone therapy. Circulation 1983;67: 45.

2 McGovern B, Garan H, Kelly E, Ruskin JN. Adverse reactions during treatment with amiodarone hydrochloride. $B M \mathcal{F}$ $1983 ; 287: 175-80$

3 Plonteux G, Heagham C, Ernowald H, Vandeghen N. Longterm hepatic tolerance of amiodarone in the clinic. Eur $\mathcal{F}$ Pharmacol 1969; 8: 369-76.

4 Stevenson RNS, Nayam TH, Davies JR. Acute hepatic dysfunction following parenteral amiodarone administration. function following parenteral
Postgrad Med $\mathcal{F}$ 1989; 65: 707-8.

5 Lupon-Roses J, Simo-Canonge R, Lu-Cortez L, PermanyerMiralda G, Allende-Mondus H. Probable early acute hepatitis with parenteral amiodarone. Clin Cardiol 1986; 9: 225-9.

6 Pye $M$, Northcote RJ, Cobbe SM. Acute hepatitis after parenteral amiodarone administration. Br Heart $\mathcal{F} 1988 ; 59$ 690-1.

7 Ruter HD. The E-Ferol syndrome solved? Side effects of drugs, Annual 12. 1988: 329. 